\title{
DISCUSSION FOLLOWING PAPER BY WOOLF
}

Schramm to Woolf: How accurate is the value $6 M_{\odot}$ which you presented for the division between stars eventually forming white dwarfs and stars becoming supernovac?

Woolf: There are several ways of estimating this number. One is the presence of a white dwarf in the Pleiades, where stars of 6-7 $M_{\odot}$ could just have evolved. The frequency of supernovae, assuming that they are stars more massive than those which become white dwarfs suggests $6 M_{\odot}$. A similar value is obtained from the frequency of planetary nebulae, assuming that they do become white dwarfs. The uncertainty in the figure is however quite large; I estimate that it lies in the range $4 M_{\odot}$ to $8 M_{\odot}$.

Arnett to Woolf: Work by S. Falk and me suggests that to obtain light curves of type II supernovae we need an extended circumstellar shell of the sort mentioned by Prof. Woolf. It would be interesting to know whether these observed objects are in early or late evolutionary stages.

Mass loss seems to be a function of mass and chemical composition. Would Prof. Woolf like to summarize his ideas on this?

Woolf: There appear to be two groups of stars with regard to chemical composition. In those with heavy element abundances greater than $1 / 20$ normal the formation of solid particles and the consequent radiation pressure on grains is very important. For lower heavy element abundance a different process is required. Similarly radiation pressure is less useful for the more massive stars but the precise dependence on mass is not clear.

Thomas: A group at Paris and Nice is studying the energetics of mass loss; in particular how is the energy stored in the region between the photosphere and the point where mass is lost. Eventually this study of atmospheric structure may provide good predictions of the rate of mass loss.

Woolf: The radiation pressure only becomes dominant in the envelope after the dust has formed and I agree that the energetics of the process are settled much lower in the atmosphere. However, I believe that the matter in the extended envelope is far too cool for it to escape, and this is why the formation of dust is crucial for mass loss.

However regardless of the physical process, the most important point for this conference is that the dust is a tracer for mass loss and allows us to estimate how much matter leaves a particular kind of star.

Thomas: I only wish to stress that mass loss will not be fully understood until the structure of the underlying star is also understood.

Paczyński: I would like to point out the existence of the so-called 'ring nebulae' 
which are known to exist around some single Population I Wolf-Rayet stars. NGC 6888 is the best studied case. The Population I W-R stars are believed to be $10 M_{\odot}$ helium stars, which were originally the cores of 20-30 $M_{\odot}$ hydrogen stars. It looks like those massive stars lost all their hydrogen rich envelopes, presumably while being red supergiants. More details may be found in the proceedings of the IAU Symposium 'W-R and early type stars' (held in Buenos Aires, 1971) and in the proceedings of the conference held at Boulder in 1968 ('Wolf-Rayet Stars', K. Gebbie and R. N. Thomas, editors).

Faulkner: The comments so far have addressed themselves to mass loss after supergiant of late (second) giant stages. It is possible to distinguish mass loss between the first giant branch and horizontal branch (as currently required by theories), e.g. by coupling composition studies with mass loss studies?

Thomas: No, not from our point of view.

Woolf: I cannot distinguish. 